\title{
Menopausal Hormone Therapy and Risk of Endometrial Cancer
}

\author{
Louise A. Brinton, Ph.D. ${ }^{1,2}$ and Ashley S. Felix, Ph.D. ${ }^{1}$ \\ ${ }^{1}$ Hormonal and Reproductive Epidemiology Branch; National Cancer Institute; Rockville, MD \\ 20852-7234
}

\section{Abstract}

Endometrial cancer is clearly a hormonally-responsive tumor, with a critical role played by estrogens unopposed by progestins. Numerous epidemiologic studies have shown substantial risk increases associated with use of unopposed estrogens, especially among thin women. This risk, however, can be reduced if progestins are added to the therapy. The manner in which progestins are prescribed is a critical determinant of risk. Most studies show that women who have ever used progestins continuously ( $>25$ days/months) are at somewhat reduced risk relative to non-users (meta-analysis relative risk, RR, based on observational studies $=0.78,95$ confidence intervals, CI, $0.72-0.86)$. The reduced risk in greatest among heavy women. In contrast, women who have ever used progestins sequentially for $<10$ days each month are at increased risk, with meta-analysis results showing on overall RR of 1.76 (1.51-2.05); in contrast, progestins given for 10-24 days/ month appear unrelated to risk $(\mathrm{RR}=1.07,0.92-1.24)$. These risks were based on varying patterns of usage, with little information available regarding how endometrial cancer risk is affected by duration of use, type and/or dose of estrogen or progestin, or mode of administration. Effects may also vary by clinical characteristics (e.g., differences for type I vs. II tumors). Further resolution of many of these relationships may be dependent on pooling data from multiple studies to derive sufficient power for subgroups of users. With changing clinical practices, it will be important for future studies to monitor a wide range of exposures and to account for divergent effects of different usage patterns.

\section{Introduction}

Endometrial cancer is the fourth most common cancer among women in the U.S., with approximately 47,000 cases diagnosed annually (1). Recognized as an hormonallyresponsive tumor, a number of risk factors have been identified that support a central causal role in endometrial cancers for both estrogen and progesterone. Many of these risk factors provide support for an etiologic hypothesis, known as the unopposed estrogen hypothesis, based on observations that exposure of the endometrium to estrogen without concomitant progesterone can stimulate endometrial cell proliferation that can increase the likelihood of

\footnotetext{
${ }^{2}$ For correspondence, contact Dr. Brinton at 6120 Executive Blvd., Rm. 5018; Phone: (301) 496-1693; Fax (301) 402-0916; brinton@nih.gov.

Publisher's Disclaimer: This is a PDF file of an unedited manuscript that has been accepted for publication. As a service to our customers we are providing this early version of the manuscript. The manuscript will undergo copyediting, typesetting, and review of the resulting proof before it is published in its final citable form. Please note that during the production process errors may be discovered which could affect the content, and all legal disclaimers that apply to the journal pertain.
} 
genetic errors and malignant transformation $(2 ; 3)$. Increased risks of endometrial cancer can result from either excessive estrogen or a deficiency in progesterone.

Several of the major risk factors for endometrial cancer, notably early ages at menarche, late ages at menopause and obesity, provide strong support for the unopposed estrogen hypothesis (4), but probably the most convincing evidence derives from extensive data linking high risks of endometrial cancer to unopposed estrogen use. Beginning in the 1960s, estrogen therapy became commonplace to help ameliorate menopausal symptoms and then became increasingly commonly prescribed to prevent such conditions as osteoporosis and heart disease. Following increases in sales of estrogens in the 1970s and rising endometrial cancer incidence rates in the 1970s (5), studies confirmed risk increases associated with estrogen use of between 2 and 10, depending on the duration of usage (6). The highest risks have been seen after 10 years of use, but it is unclear whether risk continues to increases beyond 15 years of use. In most investigations, the increased risk does not become apparent until the drugs have been used for at least 2-3 years. Most, but not all studies, have found that cessation of use is associated with a relatively rapid decrease in risk, although a number of studies have found significantly elevated risks to persist 10 or more years after last usage $(7 ; 8)$.

As a result of the recognition that unopposed estrogens were associated with such large increases in risk and that the addition of a progestin (synthetic progesterone) could counteract the proliferative effects of exogenous estrogens on the endometrium (9-12), it subsequently became commonplace for estrogens to be prescribed in conjunction with a progestin, i.e., as estrogen plus progestin therapy (EPT) — particularly for women with intact uteri. This was found to offer a benefit over unopposed estrogen therapy with respect to the development of endometrial hyperplasia (13). Nonetheless, there remains considerable uncertainty as to whether EPT is totally safe with respect to the risk of endometrial cancer. Thus, most of the recent epidemiologic studies on the effects of menopausal hormones on endometrial cancer risk have focused on the relative risks and benefits of prescribing progestins, either continuously (usually defined as $>25$ days/month) or sequentially (usually defined as $<25$ days). Some studies have also examined risks among women prescribed progestins for very short periods of time ( $<10$ days per month). Given the relatively recent advent of prescribing estrogens in conjunction with a progestin, many studies have been limited in their ability to assess the effects of long-term usage patterns, although more recent studies are beginning to bring some clarity to their associated effects.

In addition to problems with small numbers, the results from studies regarding EPT have been difficult to interpret because of varying study subject characteristics, including differences in body mass index (BMI), a factor that can modify the effects of hormones. Obese women (BMI $\geq 30 \mathrm{~kg} / \mathrm{m}^{2}$ ) have been shown to have three- to five-times the risk of endometrial cancer compared to women of normal BMI $\left(<25 \mathrm{~kg} / \mathrm{m}^{2}\right)(14 ; 15)$, reportedly stemming from increased conversion of androgens to estrogens and higher circulating concentrations of estradiol in peripheral fat tissue (16). Unopposed estrogens have been demonstrated to exert the strongest increased risks among thin or normal weight women, with obese women showing lesser hormone-associated risks, presumably reflecting a saturation effect of high circulating endogenous estrogens among obese women d\}. Less, 
however, is known about whether endometrial cancer risk among EPT users is modified by BMI, particularly as related to specific regimens or length of use of these preparations.

To resolve the effects of EPT on endometrial cancer risks, a number of recent studies have evaluated relationships, focusing on variations in risks by how the progestins are prescribed as well as on how associations are modified by a woman's BMI. The results of these recent studies are reviewed below.

\section{Effects of Continuous EPT}

Some previous observational studies have presented a confusing picture of the relationship of continuous EPT and endometrial cancer, with results ranging from no association (17-20) to increased risks (21-23) to modest decreased risks (24-26). However, more recent studies that have generally had larger samples sizes and greater statistical power to detect effects have mainly shown decreased risks (27-31). A meta-analysis of these studies, as shown in Figure 1, shows an overall relative risk (RR) of 0.81 (95\% CI 0.72-0.90) for case-control studies and $0.74(0.63-0.87)$ for cohort studies, for a combined estimate of $0.78(0.72-0.86)$. This figure is similar to the estimate of $0.88(0.75-1.03)$ derived from a smaller number of studies that was previously reported by Beral and others (28).

Most results regarding continuous EPT are from observational studies, but consistent results derive from two randomized trials in which treated women were prescribed 0.625 milligrams (mg) of conjugated equine estrogens with $2.5 \mathrm{mg}$ of medroxyprogesterone acetate. In the Women's Health Initiative (WHI) trial, women assigned to continuous EPT had a hazard ratio (HR) of 0.81 (95\% CI 0.41-1.22) compared to those assigned to placebo (32), while in the Heart and Estrogen/Progestin Therapy trial a HR of $0.25(0.05-1.18)$ was observed (33). In the WHI trial, no appreciable differences in the two arms of the trial were found in the distributions of tumor histology, stage, or grade. The findings from both trials, however, were limited by small case numbers (58 and 10 respectively) and short exposure durations.

Although there are consistent findings from both observational studies and trials of a reduced risk of endometrial cancer associated with continuous EPT use, uncertainty exists as to how risk is affected by duration of use. One analysis that combined results from a number of case-control studies in a single geographic area, namely Seattle, showed persistent decreases with long-term use, defined as 10 or more years (34), consistent with results from a Finnish record linkage study (29). In contrast, in a recent U.S. cohort, reduced risks that were seen for short-term EPT use ( $<10$ years: RR: $0.53,95 \%$ CI 0.39-0.73) appeared to diminish with longer term use ( $\geq 10$ years: RR: $0.98,95 \%$ CI $0.63-1.54)(31)$. In yet another investigation, not association of risk was seen for ever use, but there was evidence of increased risks among the long-term users (23). This finding, however, may have mainly reflected that longer term usage was more prevalent among the thinner women. An additional reason for inconsistencies across studies could reflect unstable estimates due to small numbers. Thus, the issue of how long-term use of continuous EPT therapy affects endometrial cancer risk needs pursuit by additional research. 
As with other hormone usage patterns, relationships appear highly dependent on interactions with other host indicators of endogenous estrogen levels (most notably BMI). Most studies $(23 ; 31 ; 34 ; 35)$ have shown the greatest reduction associated with continuous EPT use among heavier women. In some studies, the reduction has been profound. For instance, in a recent U.S. cohort study, the relative risk associated with use of continuous EPT was 0.34 (95\% CI $0.20-0.58)$ among women with a BMI of 30 or greater, as compared with $1.14(0.71-1.83)$ among those with a BMI less than 25 (31). These results provide growing support for exogenous progestins opposing the proliferative effects of endogenous estrogens.

Although it appears clear that BMI can substantially alter the effects of continuous EPT, studies to date have been limited in their ability to assess how obesity modifies effects of usage patterns. Hypothetically, obese women with long-term usage would be those who would benefit most relative to non-usage, but specific studies on this issue are not currently available.

\section{Effects of Sequential EPT}

Most studies have linked sequential EPT use with an increased endometrial cancer risk relative to non-use. This association has been observed in case-control $(20 ; 26 ; 35)$, cohort $(18 ; 21 ; 27)$ and record linkage (29) studies. Only a few studies have failed to demonstrate a relationship between use of sequential EPT use and endometrial cancer risk, possibly reflecting either small numbers of hormone users or longer exposures to progestin use per cycle $(17 ; 30 ; 36)$. Beral's meta-analysis (28) of many of these studies indicated an overall RR of 1.14 (95\% CI 1.01-1.28) associated with sequential EPT use.

In most of the investigations showing positive relationships, the increased risks related to sequential usage appeared to be driven by higher risks related to current or long duration use. Further, in several studies, in line with the notion that progestins ameliorate the adverse effects of estrogens on endometrial tissue $(11 ; 12)$, the increased risk appeared to be related to the length of time that estrogens were unopposed by progestins $(18 ; 23 ; 35)$. Particularly high risks have been noted when long-cycle EPT is involved, such as has occurred in several European countries, including Finland (29), in which progestogens are used for only 10-14 days a month every three months. In U.S. studies, higher risks have been noted when progestins are prescribed for fewer than 10 days each months, with a meta-analysis of available studies demonstrating an overall RR of 1.76 (95\% CI 1.51-2.05) (Figure 2). In contrast, no overall excess risk has been observed when progestins have been given for 10 or more days each month (meta-analysis RR=1.07, 95\% CI 0.92-1.24) (Figure 3). Although this is reassuring in terms of safety, data are currently lacking on whether risk remains nonelevated when long-duration use of the regimen is involved.

Although sequential therapy appears to be associated with a higher risk of endometrial cancer than continuous EPT use, in several recent studies it has been shown to be associated with a smaller increased risk than unopposed estrogen hormone therapy $(26 ; 27)$. This finding supports the notion that the addition of a progestin to hormone therapy weakens the excess risk of endometrial cancer associated with unopposed estrogen use. 
Similar to what has been seen for unopposed estrogens and continuous EPT, effects of sequential usage appears to be modified by body mass. However, in contrast to stronger protective effects of continuous EPT use in heavy women, sequential EPT use appears to be associated with the strongest increased risks among thin women. This has been seen for current users in the Million Women Study (28) and for long-term users in a case-control study in Washington state (35). In a recent analysis, conducted within the NIH-AARP cohort, sequential EPT use was associated with a RR of 2.53 (95\% CI 1.71-3.74) among thin women, as compared to $0.76(0.46-1.27)$ among obese women (31).

\section{Absolute Risk Patterns Associated with Different Hormone Usage Patterns}

Since it is clear that a proper interpretation of the effect of different patterns of menopausal hormone usage is dependent on a consideration of intervening effects of BMI, the most informative studies are those that present absolute risks, based on the derivation of agestandardized incidence rates $(28 ; 31)$. This is due to the over-riding importance of obesity as a predictor of endometrial cancer risk. Although relative risks support the strongest apparent protective effects of combination EPT among obese women, the highest risks still prevail among obese women. Thus, in the large NIH-AARP study, incidence rates among continuous EPT users rose from 6.0 per 10,000 women for normal weight women to 8.8 for overweight women to 11.9 for obese women (31) —with the highest risks seen among nonhormone users (35.9). Comparable rates for sequential therapy were 15.4, 13.2 and 29.0. In thin women, however, both sequential and continuous EPT users had higher incidence rates than non-users (who had an incidence rate of 5.5), although somewhat different trends have been observed in the two studies that have evaluated these absolute risks (28;31). This most likely reflects complicating effects of other usage patterns (e.g., duration of use), which should be evaluated in future studies.

\section{Modifying Effects of Other Endometrial Cancer Risk Factors on Hormone Associations}

BMI clearly impacts the effects of different hormone regimens, but less is known regarding whether other endometrial cancer risk factors can have modifying influences. Early studies presented conflicting results regarding whether the effects of unopposed estrogens were modified by other endometrial cancer risk factors, including cigarette smoking, chronic disease histories (e.g., diabetes), or use of oral contraceptives (7;37;38). Few similar evaluations have been undertaken with respect to EPT regimens. In the European Prospective Investigation into Cancer (EPIC) study, higher EPT-associated risks were found among older women and ever smokers (27). The Million Women Study also confirmed somewhat higher risks associated with sequential EPT among older women, but no variation by smoking status. In this large study, the only factor that appeared to modify the relationship of continuous EPT was BMI (28). Potential interactions by age (and smoking) could reflect stronger estrogenic effects in women with lower circulating estrogen levels, but alternatively these stronger purported associations could also reflect longer durations of use. These interactions are also difficult to assess given the overriding impact that BMI has on hormone relationships. 


\section{Type and Dose of Estrogens and Progestins Prescribed}

Studies that have found discrepant responses of endometrial tissue to different estrogens and progestins (39) have prompted several investigators to assess relationships according to type of hormones prescribed. Results from some early studies of unopposed estrogens suggesting that higher risks might be associated with use of conjugated as compared with synthetic estrogens (such as estradiol) (6) have not been subsequently confirmed (26). However, there are now extensive data supporting an important role for estrogen dose in contributing to higher observed risks $(6 ; 26)$.

Studies regarding effects of type of progestin have provided less clear results. Weiderpass et al. (26) found that progesterone-derived progestins prescribed sequentially seem to lead to higher risks than testosterone-derived progestins. Allen and others (27) found that use of micronized progesterones (mainly sequentially) were associated with increased risk, whereas testosterone or progesterone-derived derivatives were not. Two other studies $(28 ; 29)$, however, noted no difference in risk between sequential use of norithisterone and medroxprogesterone acetate. These discrepant results, however, may have reflected confounding effects by the type and dose of estrogen used, or duration of progestin use.

Few studies have evaluated the effects of progestin dose. Although one case-control study reported the highest risk among continuous users of $10 \mathrm{mg}$ per day of MPA (22), an effect of progestin dose was not found among varied regimen users in another study (40). The relationship is difficult to evaluate given that it is common practice to increase the progestin dose in hormone users who develop vaginal bleeding, which is often an early symptom of endometrial cancer.

\section{Mode of Administration}

Despite increasing numbers of women being administered hormones in the form of transdermal patches, and many women having previously received injectable forms of hormones, there has been little evaluation of associated risks. One Finnish study found no difference in effects of oral versus transdermal routes of administration of either continuous or sequential EPT (41), although whether these results are relevant to U.S. women is questionable given that the predominant form of estrogen used in Finland is estradiol rather than conjugated estrogens (which are more commonly prescribed in the U.S). A subsequent study in Finland (29) evaluated effects of estradiol plus levonorgestrel-releasing intrauterine devices, and found them to be associated with a significant reduction in endometrial cancer risk $(\mathrm{RR}=0.39,95 \%$ CI $0.17-0.88)$.

\section{Tibolone}

Although not currently licensed in the U.S., several European countries are currently prescribing tibolone, a synthetic steroid with estrogenic, progestagenic and androgenic properties, as a menopausal hormone treatment. Several early studies suggested an increased risk associated with use of this drug (42;43), but more convincing evidence now derives from the large U.K. Million Women Study (28), in which women whose last hormone usage consisted of tibolone had a relative risk of 1.79 (95\% CI 1.43-2.25) compared to non-users. 
The risk was higher among women who reported longer-term usage of these preparations and those who had a BMI of 25 or less $(\mathrm{RR}=2.99$, 95\% CI 2.08-4.30). The association also remained when analyses were restricted to women who were likely to have used tibolone exclusively. Further confirmation of a potentially adverse effect of tiblolone on endometrial cancer risk was seen in the EPIC study, which noted nearly a 3-fold increased risk associated with usage (27). A recent Finnish study did not observe any increase in endometrial cancer risk associated with tibolone, but the prevalence of exposure was low and small numbers were involved (29).

\section{Variation in Hormone Associations by Endometrial Cancer Clinical Parameters}

A number of early studies of unopposed estrogens suggested that tumors associated with unopposed estrogen use generally demonstrate favorable characteristics, including earlier stage at diagnosis, lower grade, and fewer instances of myometrial invasion $(6 ; 7 ; 44)$. Estrogen users also tended to be younger at diagnosis than patients who had not used estrogens and the tumors were more frequently accompanied by hyperplasia or adenomyosis (45). Although it was suggested that these observations could indicate that some advanced endometrial hyperplasias were being diagnosed as endometrial carcinomas, the relationship between estrogen use and increased endometrial cancer risk persists when such cases are eliminated.

In line with these observations, early debate focused on the extent to which these relationships might reflect detection bias, whereby estrogen-induced bleeding would lead to the diagnosis of cancer among women who would otherwise remain asymptomatic.

However, there are now a number of studies providing data to counteract this argument, namely that 1) estrogen associations persist even among women who do not present with uterine bleeding, 2) only a small number of women appear to go undiagnosed on the basis of necropsy studies, and 3) associations are strongest for long-term users, which is opposite to what would be hypothesized if detection biases were operating. Although the theory of detection bias as an explanation for the link between hormone use and increased endometrial cancer has now largely been rebuked, reasons for hormones being more strongly associated with lower grade and stage tumors remains unclear. Pike and others (20) have suggested that this may reflect increased surveillance, but studies to specifically evaluate this are needed.

Despite evidence that unopposed estrogens have stronger effects for early stage cancers, few studies have evaluated relationships according to other regimens of use. Pike et al (20) observed that all forms of menopausal hormone use showed decreasing risks with increasing stages of disease at diagnosis. The most striking difference was seen for short-term progestin (<10 days) sequential use, where the odds ratio (OR) associated with use was 3.07 (1.934.89) for stage IA cancers as compared with 1.05 0.43-2.58) for IC+ tumors. Similar decreasing trends in risks with stage were also seen for long-term progestin sequential use as well as continuous EPT. Similar to their trends observed for unopposed estrogen users, Weiderpass and others (26) found risks associated with combined therapy to higher when there was either increasing differentiation of the endometrial neoplasias or myometrial infiltration, but these relationships were confined to cyclic and not continuous treatment. 
In addition to hormone associations varying by disease stage, there has been interest in assessing associations by histologic subtype, given increasing recognition that endometrial cancer is a heterogeneous disease and that some subtypes are more influenced by hormonal factors. Under a scheme first proposed by Bokhman (46), two broad types of endometrial cancer have been investigated, with Type I tumors (or low-grade endometrioid tumors) being more influenced by hormonal factors than poorer prognosis Type II tumors. Studies are just beginning to assess epidemiologic differences between these two broad classes of tumors, and there may be additional heterogeneity that is still to be recognized. One recent meta-analysis concluded that there is no significant variation in hormone effects by histologic subtypes (14), but another large cohort study noted higher hormone-associated risks for Type I than II cancers, albeit without the ability to assess interactions by type of hormones prescribed (47).

\section{Menopausal Hormones and Endometrial Cancer Survival}

Survival outcomes related to menopausal hormone therapy have mainly been explored in the context of unopposed estrogen use. To date, the evidence has been conflicting: studies have reported no association (48-52), improved survival (53-56) and worse survival (57) among users of unopposed estrogen compared to non-users. Among studies reporting improved survival associated with prior unopposed estrogen use, only three adjusted for tumor characteristics $(53 ; 55 ; 58)$. Importantly, users of menopausal hormones who develop endometrial cancer tend to have earlier stage and lower grade tumors, which may explain the relationship between menopausal hormones and improved survival. Furthermore, in many of these studies, small numbers of deaths among exposed cases limited the power to assess relationships.

Other formulations of exogenous hormone use, specifically sequential and continuous EPT, have been explored infrequently in relation to survival among endometrial cancer patients. Using data from a population-based Swedish cohort, Schairer and colleagues (55) calculated Standardized Mortality Ratios (SMRs) associated with use of sequential EPT or unopposed estrogen regimens. A lower risk of endometrial cancer-specific death was noted among sequential EPT users, however this finding was non-significant due to only 2 deaths occurring in this subgroup (SMR: 0.6, 95\% CI 0.1, 2.1).

Most recently, Orgeas and colleagues (54) examined the role of various menopausal hormone regimens, including sequential and continuous EPT, on tumor characteristics and overall survival outcomes in a Swedish register study. Relative excess hazard ratios (RER) showed improved survival for users of any form of hormone therapy (RER CI $=0.40,95 \%$ CI 0.16-0.97) and any form of estrogen therapy, including regimens with or without progestin (RER: $0.38,0.15-0.99$ ). These estimates, however, were only adjusted for age and calendar year, despite the significant associations observed between any hormone or estrogen use and well-differentiated, less invasive tumors.

Given the lack of studies evaluating prediagnosis use of specific hormone regimens and endometrial cancer mortality, future studies should explore this relationship in cohorts with 
large numbers of exposed cases and adjustment for potential confounding factors, including grade and stage.

\section{Summary and Conclusions}

Much of what is now known regarding underlying biologic mechanisms involved in endometrial carcinogenesis derives from the study of the effects of menopausal hormones. Substantial risk increases associated with unopposed estrogens provide firm support for a critical role for estrogens unopposed by progestins. This risk, however, can be effectively reduced if progestins are added to the therapy. Emerging evidence, however, suggests that how these progestins are prescribed is crucial to the safety of prescribing estrogens.

Although recent research has brought much clarity to this important question, a number of issues remain unresolved, particularly as related to long-term usage patterns. For instance, it remains controversial whether the reduced risk seen in most studies for continuous EPT persists for long-term users. The risk experienced by long-term sequential EPT users is also uncertain. It is clear, however, that a proper interpretation of effects is dependent on considering potential modifying effects of other endometrial cancer risk factors. Although BMI is accepted as an important factor to consider, it is also possible that other factors known to operate through hormonal mechanisms, such as oral contraceptive use or cigarette smoking, could also have intervening effects. Further investigations of these potential interactions are needed.

It will also be important for future studies to appreciate the considerable etiologic heterogeneity that exists within endometrial cancer, and to assess hormone relationships according to different clinical parameters, such as stage and histology. There is growing appreciation that risk factors may vary between Type I and Type II endometrial cancers, but it is also possible that there may be additional heterogeneity according to other defined clinical parameters.

To fully address these issues, it may be necessary to pool data from multiple studies. A number of such consortial efforts are ongoing, which should bring further clarity to the effects of hormones as they have been and are currently prescribed. With changing clinical practices and different agents being prescribed, it will be important for future studies to monitor a wide range of exposures, and to appreciate the considerable complexity that previous studies have shed on the issue of endometrial hormonal carcinogenesis.

\section{Reference List}

1. Howlader, N.; Noone, AM.; Krapcho, M.; Neyman, N.; Aminou, R.; Altekruse, SF., et al. SEER Cancer Statistics Review, 1975-2009. National Cancer Institute; Bethesda, MD: Nov 1. 2011 p. 412http://seer.cancer.gov/csr/1975_2009_pops09Ref Type: Electronic Citation

2. Akhmedkhanov A, Zeleniuch-Jacquotte A, Toniolo P. Role of exogenous and endogenous hormones in endometrial cancer: review of the evidence and research perspectives. Ann N Y Acad Sci. 2001; 943:296-315. [PubMed: 11594550]

3. Key TJ, Pike MC. The dose-effect relationship between 'unopposed' oestrogens and endometrial mitotic rate: its central role in explaining and predicting endometrial cancer risk. Br J Cancer. 1988; 57(2):205-12. [PubMed: 3358913] 
4. Cramer DW. The epidemiology of endometrial and ovarian cancer. Hematol Oncol Clin North Am. 2012; 26(1):1-12. [PubMed: 22244658]

5. Weiss NS, Szekely DR, Austin DF. Increasing incidence of endometrial cancer in the United States. N Engl J Med. 1976; 294(23):1259-62. [PubMed: 1264151]

6. Grady D, Gebretsadik T, Kerlikowske K, Ernster V, Petitti D. Hormone replacement therapy and endometrial cancer risk: a meta-analysis. Obstet Gynecol. 1995; 85(2):304-13. [PubMed: 7824251]

7. Brinton LA, Hoover RN. Estrogen replacement therapy and endometrial cancer risk: unresolved issues. The Endometrial Cancer Collaborative Group. Obstet Gynecol. 1993; 81(2):265-71. [PubMed: 8380913]

8. Green PK, Weiss NS, McKnight B, Voigt LF, Beresford SA. Risk of endometrial cancer following cessation of menopausal hormone use (Washington, United States). Cancer Causes Control. 1996; 7(6):575-80. [PubMed: 8932917]

9. Campbell S, McQueen J, Minardi J, Whitehead MI. The modifying effect of progestogen on the response of the post-menopausal endometrium to exogenous oestrogens. Postgrad Med J. 1978; 54 (Suppl 2):59-64. [PubMed: 740582]

10. Lethaby A, Suckling J, Barlow D, Farquhar CM, Jepson RG, Roberts H. Hormone replacement therapy in postmenopausal women: endometrial hyperplasia and irregular bleeding. Cochrane Database Syst Rev. 2004; (3):CD000402. [PubMed: 15266429]

11. Whitehead MI, Campbell S, Dyer G, Collins WP, Pryse-Davies J, Ryder TA, et al. Progestogen modification of endometrial histology in menopasual women. Br Med J. 1978; 2(6152):1643-4. [PubMed: 728765]

12. Whitehead MI, King RJ, McQueen J, Campbell S. Endometrial histology and biochemistry in climacteric women during oestrogen and oestrogen/progestogen therapy. J R Soc Med. 1979; 72(5):322-7. [PubMed: 552525]

13. Furness S, Roberts H, Marjoribanks J, Lethaby A. Hormone therapy in postmenopausal women and risk of endometrial hyperplasia. Cochrane Database Syst Rev. 2012; 8:CD000402. [PubMed: 22895916]

14. Crosbie EJ, Zwahlen M, Kitchener HC, Egger M, Renehan AG. Body mass index, hormone replacement therapy, and endometrial cancer risk: a meta-analysis. Cancer Epidemiol Biomarkers Prev. 2010; 19(12):3119-30. [PubMed: 21030602]

15. Renehan AG, Tyson M, Egger M, Heller RF, Zwahlen M. Body-mass index and incidence of cancer: a systematic review and meta-analysis of prospective observational studies. Lancet. 2008; 371(9612):569-78. [PubMed: 18280327]

16. Lukanova A, Lundin E, Zeleniuch-Jacquotte A, Muti P, Mure A, Rinaldi S, et al. Body mass index, circulating levels of sex-steroid hormones, IGF-I and IGF-binding protein-3: a cross-sectional study in healthy women. Eur J Endocrinol. 2004; 150(2):161-71. [PubMed: 14763914]

17. Jain MG, Rohan TE, Howe GR. Hormone replacement therapy and endometrial cancer in Ontario, Canada. J Clin Epidemiol. 2000; 53(4):385-91. [PubMed: 10785569]

18. Karageorgi S, Hankinson SE, Kraft P, De VI. Reproductive factors and postmenopausal hormone use in relation to endometrial cancer risk in the Nurses' Health Study cohort 1976-2004. Int J Cancer. 2010; 126(1):208-16. [PubMed: 19551854]

19. Lacey JV Jr, Leitzmann MF, Chang SC, Mouw T, Hollenbeck AR, Schatzkin A, et al. Endometrial cancer and menopausal hormone therapy in the National Institutes of Health-AARP Diet and Health Study cohort. Cancer. 2007; 109(7):1303-11. [PubMed: 17315161]

20. Pike MC, Peters RK, Cozen W, Probst-Hensch NM, Felix JC, Wan PC, et al. Estrogen-progestin replacement therapy and endometrial cancer. J Natl Cancer Inst. 1997; 89(15):1110-6. [PubMed: 9262248]

21. Lacey JV Jr, Brinton LA, Lubin JH, Sherman ME, Schatzkin A, Schairer C. Endometrial carcinoma risks among menopausal estrogen plus progestin and unopposed estrogen users in a cohort of postmenopausal women. Cancer Epidemiol Biomarkers Prev. 2005; 14(7):1724-31. [PubMed: 16030108]

22. Newcomb PA, Trentham-Dietz A. Patterns of postmenopausal progestin use with estrogen in relation to endometrial cancer (United States). Cancer Causes Control. 2003; 14(2):195-201. [PubMed: 12749724] 
23. Razavi P, Pike MC, Horn-Ross PL, Templeman C, Bernstein L, Ursin G. Long-term postmenopausal hormone therapy and endometrial cancer. Cancer Epidemiol Biomarkers Prev. 2010; 19(2):475-83. [PubMed: 20086105]

24. Chubak J, Doherty JA, Cushing-Haugen KL, Voigt LF, Saltzman BS, Hill DA, et al. Endometrial cancer risk in estrogen users after switching to estrogen-progestin therapy. Cancer Causes Control. 2007; 18(9):1001-7. [PubMed: 17653829]

25. Hill DA, Weiss NS, Beresford SA, Voigt LF, Daling JR, Stanford JL, et al. Continuous combined hormone replacement therapy and risk of endometrial cancer. Am J Obstet Gynecol. 2000; 183(6): 1456-61. [PubMed: 11120510]

26. Weiderpass E, Adami HO, Baron JA, Magnusson C, Bergstrom R, Lindgren A, et al. Risk of endometrial cancer following estrogen replacement with and without progestins. J Natl Cancer Inst. 1999; 91(13):1131-7. [PubMed: 10393721]

27. Allen NE, Tsilidis KK, Key TJ, Dossus L, Kaaks R, Lund E, et al. Menopausal hormone therapy and risk of endometrial carcinoma among postmenopausal women in the European Prospective Investigation Into Cancer and Nutrition. Am J Epidemiol. 2010; 172(12):1394-403. [PubMed: 20961969]

28. Beral V, Bull D, Reeves G. Endometrial cancer and hormone-replacement therapy in the Million Women Study. Lancet. 2005; 365(9470):1543-51. [PubMed: 15866308]

29. Jaakkola S, Lyytinen HK, Dyba T, Ylikorkala O, Pukkala E. Endometrial cancer associated with various forms of postmenopausal hormone therapy: a case control study. Int J Cancer. 2011; 128(7):1644-51. [PubMed: 21280035]

30. Strom BL, Schinnar R, Weber AL, Bunin G, Berlin JA, Baumgarten M, et al. Case-control study of postmenopausal hormone replacement therapy and endometrial cancer. Am J Epidemiol. 2006; 164(8):775-86. [PubMed: 16997897]

31. Trabert B, Wentzensen N, Yang HP, Sherman ME, Hollenbeck AR, Park Y, et al. Is estrogen plus progestin menopausal hormone therapy safe with respect to endometrial cancer risk? Int J Cancer. 2012

32. Anderson GL, Judd HL, Kaunitz AM, Barad DH, Beresford SA, Pettinger M, et al. Effects of estrogen plus progestin on gynecologic cancers and associated diagnostic procedures: the Women's Health Initiative randomized trial. JAMA. 2003; 290(13):1739-48. [PubMed: 14519708]

33. Hulley S, Furberg C, Barrett-Connor E, Cauley J, Grady D, Haskell W, et al. Noncardiovascular disease outcomes during 6. 8 years of hormone therapy: Heart and Estrogen/progestin Replacement Study follow-up (HERS II). JAMA. 2002; 288(1):58-66. [PubMed: 12090863]

34. Phipps AI, Doherty JA, Voigt LF, Hill DA, Beresford SA, Rossing MA, et al. Long-term use of continuous-combined estrogen-progestin hormone therapy and risk of endometrial cancer. Cancer Causes Control. 2011; 22(12):1639-46. [PubMed: 21909949]

35. Doherty JA, Cushing-Haugen KL, Saltzman BS, Voigt LF, Hill DA, Beresford SA, et al. Longterm use of postmenopausal estrogen and progestin hormone therapies and the risk of endometrial cancer. Am J Obstet Gynecol. 2007; 197(2):139-7. [PubMed: 17689625]

36. Persson I, Adami HO, Bergkvist L, Lindgren A, Pettersson B, Hoover R, et al. Risk of endometrial cancer after treatment with oestrogens alone or in conjunction with progestogens: results of a prospective study. BMJ. 1989; 298(6667):147-51. [PubMed: 2538173]

37. Newcomer LM, Newcomb PA, Trentham-Dietz A, Storer BE. Hormonal risk factors for endometrial cancer: modification by cigarette smoking (United States). Cancer Causes Control. 2001; 12(9):829-35. [PubMed: 11714111]

38. Rubin GL, Peterson HB, Lee NC, Maes EF, Wingo PA, Becker S. Estrogen replacement therapy and the risk of endometrial cancer: remaining controversies. Am J Obstet Gynecol. 1990; 162(1): 148-54. [PubMed: 2301483]

39. Jondet M, Maroni M, Yaneva H, Brin S, Peltier-Pujol F, Pelissier C. Comparative endometrial histology in postmenopausal women with sequential hormone replacement therapy of estradiol and, either chlormadinone acetate or micronized progesterone. Maturitas. 2002; 41(2):115-21. [PubMed: 11836042] 
40. Reed SD, Voigt LF, Beresford SA, Hill DA, Doherty JA, Weiss NS. Dose of progestin in postmenopausal-combined hormone therapy and risk of endometrial cancer. Am J Obstet Gynecol. 2004; 191(4):1146-51. [PubMed: 15507934]

41. Jaakkola S, Lyytinen H, Pukkala E, Ylikorkala O. Endometrial cancer in postmenopausal women using estradiol-progestin therapy. Obstet Gynecol. 2009; 114(6):1197-204. [PubMed: 19935019]

42. de Vries CS, Bromley SE, Thomas H, Farmer RD. Tibolone and endometrial cancer: a cohort and nested case-control study in the UK. Drug Saf. 2005; 28(3):241-9. [PubMed: 15733028]

43. Yazigi R, Sahid S, Contreras L, Rodriguez T. Carcinoma of the endometrium in patients treated with tibolone. Gynecol Oncol. 2004; 93(2):568-70. [PubMed: 15099984]

44. Shapiro JA, Weiss NS, Beresford SA, Voigt LF. Menopausal hormone use and endometrial cancer, by tumor grade and invasion. Epidemiology. 1998; 9(1):99-101. [PubMed: 9430277]

45. Elwood JM, Boyes DA. Clinical and pathological features and survival of endometrial cancer patients in relation to prior use of estrogens. Gynecol Oncol. 1980; 10(2):173-87. [PubMed: 7461483]

46. Bokhman JV. Two pathogenetic types of endometrial carcinoma. Gynecol Oncol. 1983; 15(1):107. [PubMed: 6822361]

47. Yang HP, Wentzensen N, Trabert B, Gierach GL, Felix AS, Gunter MJ, et al. Endometrial cancer risk factors by two main histologic subtypes in the NIH-AARP Diet and Health Study. Am J Epidemiol. 2012 Ref Type: In Press.

48. Ettinger B, Golditch IM, Friedman G. Gynecologic consequences of long-term, unopposed estrogen replacement therapy. Maturitas. 1988; 10(4):271-82. [PubMed: 3226337]

49. Lafferty FW, Helmuth DO. Post-menopausal estrogen replacement: the prevention of osteoporosis and systemic effects. Maturitas. 1985; 7(2):147-59. [PubMed: 4033448]

50. Paganini-Hill A, Ross RK, Henderson BE. Endometrial cancer and patterns of use of oestrogen replacement therapy: a cohort study. Br J Cancer. 1989; 59(3):445-7. [PubMed: 2930713]

51. Petitti DB, Perlman JA, Sidney S. Noncontraceptive estrogens and mortality: long-term follow-up of women in the Walnut Creek Study. Obstet Gynecol. 1987; 70(3 Pt 1):289-93. [PubMed: 3627576]

52. Robboy SJ, Bradley R. Changing trends and prognostic features in endometrial cancer associated with exogenous estrogen therapy. Obstet Gynecol. 1979; 54(3):269-77. [PubMed: 471366] 


\section{Highlights}

Endometrial cancer risk is substantially increased among users of unopposed estrogens, with further enhancements among thin women

The addition of progestins for $>25$ days/month reduces risk, to levels even somewhat lower than that of non-hormone users

Users of progestins for $<10$ days/month remain at an elevated risk 


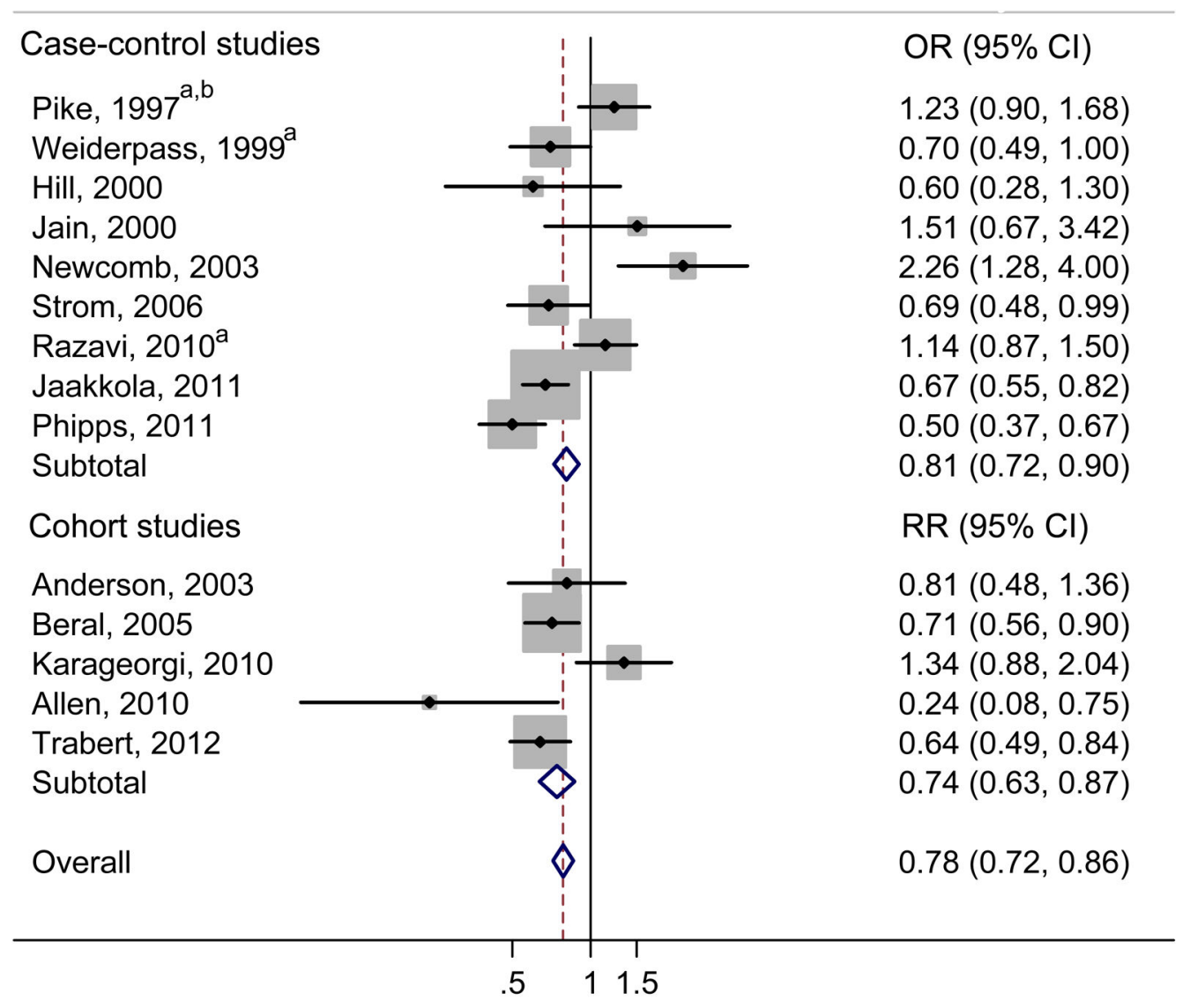

Figure 1.

Summary of key studies examining the association between continuous-combined menopausal hormone therapy and endometrial cancer risk

a. Odds ratios and $95 \%$ confidence intervals were calculated across duration categories using the inverse variance method b. Variance for the pooled estimate was calculated using the formula: $1 / a+1 / b+1 / c+1 / d$ 


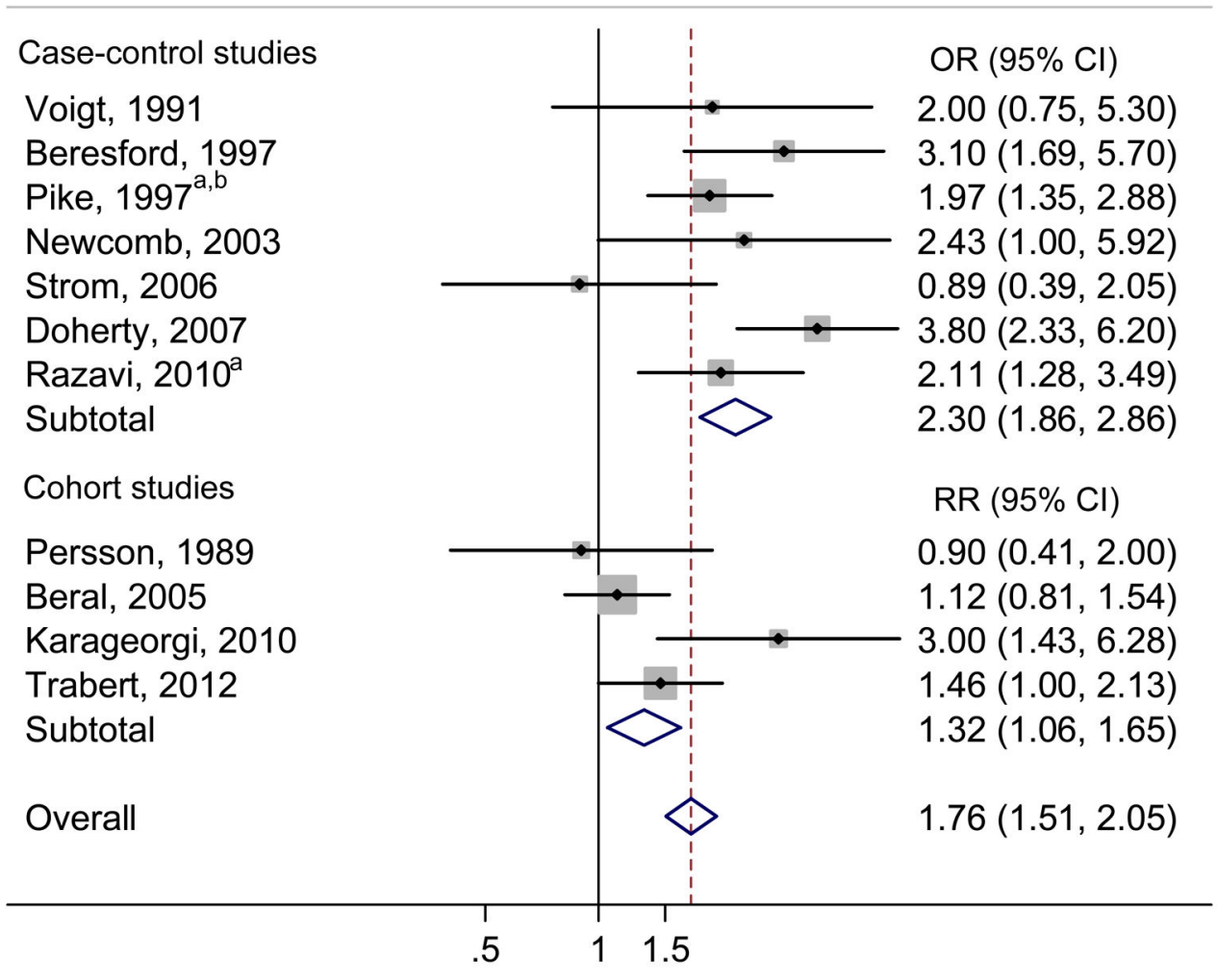

Figure 2.

Summary of key studies examining the association between sequential menopausal hormone therapy ( $<10$ days of progestin/ month) and endometrial cancer risk

a. Odds ratios and $95 \%$ confidence intervals were calculated across duration categories using the inverse variance method b. Variance for the pooled estimate was calculated using the formula: $1 / a+1 / b+1 / c+1 / d$ 


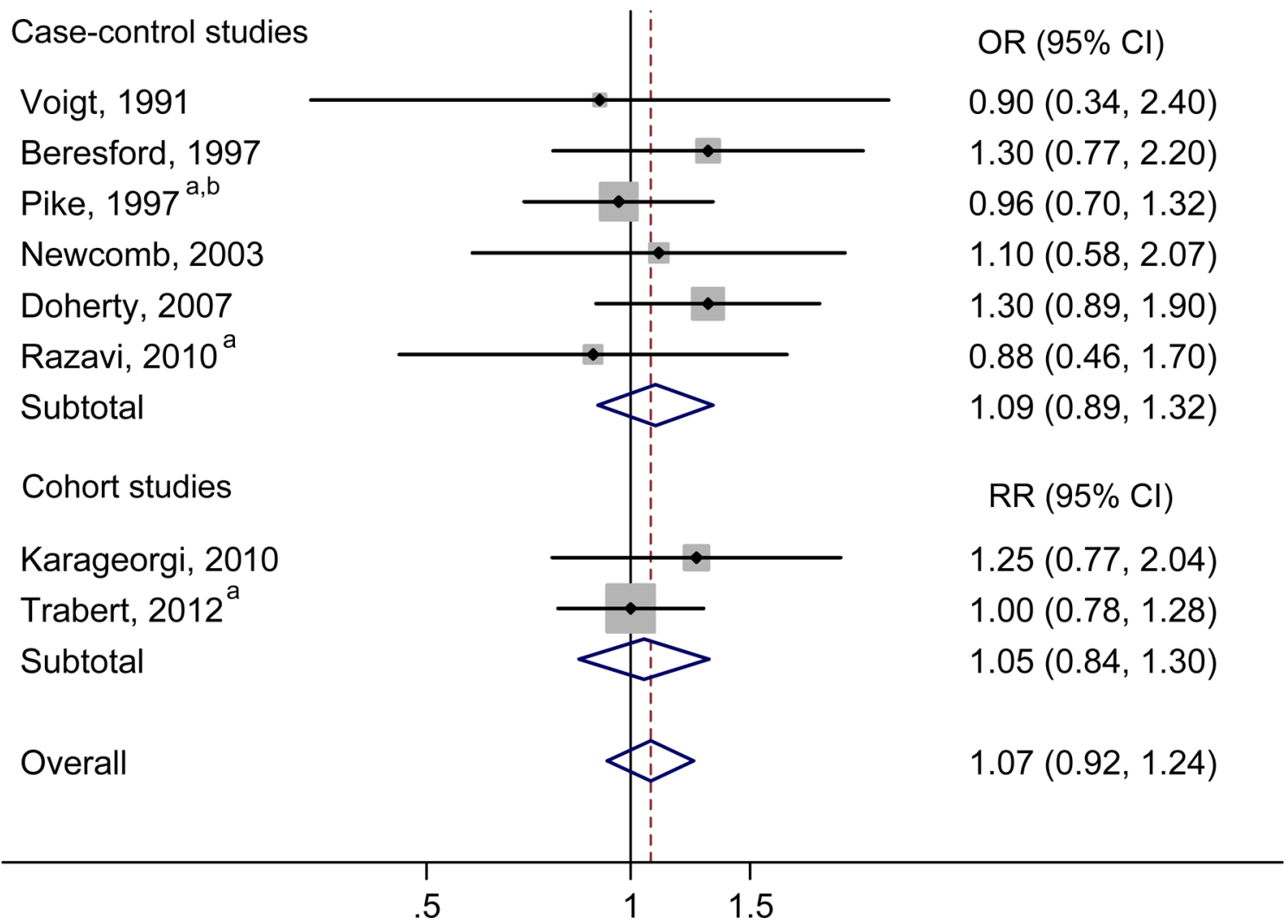

Figure 3.

Summary of key studies examining the association between sequential menopausal hormone therapy ( $\geq 10$ days of progestin/ month) and endometrial cancer risk

a. Odds ratios and $95 \%$ confidence intervals were calculated across duration categories using the inverse variance method

b. Variance for the pooled estimate was calculated using the formula: $1 / a+1 / b+1 / c+1 / d$

* Studies categorizing days of progestin as 10-14 not included (Jaakola et al., Allen et al., Weiderpass et al. 